\title{
In Treating Glucocorticoids Induced Osteoporosis in Temporomandibular Joint of Albino Rats; Which are More Effective Microvesicles or Mesenchymal Stem Cells?
}

\section{Original Article}

\author{
Rehab A Abdel Moneim ${ }^{1,2}$, Abeer Mostafa ${ }^{3}$, Marwa MS Abbass ${ }^{1}$ \\ Oral Biology Department, Faculty of Dentistry, ${ }^{1}$ Cairo University, ${ }^{2}$ Future University \\ ${ }^{3}$ Medical Biochemistry and Molecular Biology, Faculty of Medicine, Cairo University
}

\begin{abstract}
Background: Osteoporosis is a systemic skeletal disorder that increases bone fragility and risk of fractures. Current medications for treating osteoporosis are associated with osteonecrosis due to their anti-angiogenic effect that leads to ischaemic and necrotic changes.

Aim: The present research compared two new approaches in treating glucocorticoids induced osteoporosis in temporomandibular joints (TMJs) of albino rats; Microvesicles (MVs) and Mesenchymal stem cells (MSCs).

Materials and Methods: 39 adult female albino rats were divided into 3 groups. All rats were intraperitoneally injected once daily throughout the experimental period (60 days); 13 rats with $9 \%$ saline and 26 rats with dexamethasone $(200 \mu \mathrm{g} / 100 \mathrm{~g}$ body weight). After 30 days; the first group (control group) received a local injection of $1 \mathrm{ml}$ PBS in TMJs of both sides. Half the dexamethasone injected rats were locally injected in the right side TMJs with $1 \times 10^{7} / \mathrm{ml} / \mathrm{week}$ MVs (Microvesicles group). The remaining rats (Stem cells group) received 1 million MSCs/ ml/week. In the later 2 groups, the left side TMJs received $1 \mathrm{ml}$ PBS injection (untreated sides). The osteogenic potential was examined histologically, by western blotting, ELISA and RT-PCR.

Results: Histologically; both sides from MSCs group exhibited abnormal configuration of the joint's anatomy while a marked improvement of bone architecture in the microvesicles treated TMJs was detected. Treated sides in both groups (MVs, MSCs) showed a significant increase in osteogenic markers (ALP, BMP and RUNX-2) and a decrease in inflammatory markers (IL-1 $\beta$, TNF- $\alpha$, IL-6) as well as in RANKL expression as compared to untreated ones.

Conclusion: Microvesicles treated TMJs showed an enhanced pattern of bone regeneration confirmed by the higher mean bone area \% as compared to MSCs group. On the contrary, MSCs treated TMJs showed a significantly elevated level of osteogenic markers; yet the osteogenic reactivity was very aggressive that caused deformity to the TMJ architecture.
\end{abstract}

Received: 13 December 2019, Accepted: 08 January 2020

Key Words: Dexamethasone, mesenchymal stem cells, microvesicles, osteoporosis, TMJ.

Corresponding Author: Marwa Magdy Saad Abbass, PhD, Oral Biology Department, Faculty of Dentistry, Cairo University, Egypt, Tel.: +20 1119443811, E-mail: marwa.magdy@dentistry.cu.edu.eg

ISSN: $1110-0559$, Vol. 43, No.3

\section{INTRODUCTION}

Osteoporosis is a multifactorial, skeletal disease characterized by a decreased bone mineral density (BMD) and distortion of the bone tissue microarchitectural structure; leading to increased risk of fracture and loss of mechanical strength ${ }^{[1]}$. Osteoporosis is a silent disease; manifested only in a low bone density, until fracture occurs $^{[2]}$. The spine, hip, forearm and proximal humerus are the common sites of fracture. Fractures at the hip are blamed for the greatest morbidity and mortality ${ }^{[3]}$.

Osteoporosis has a direct correlation with oral and dental health. The disease might be associated with a lowered bone density of the jaws, periodontal disease, tooth loss, inability to make functional dentures and temporomandibular disorders ${ }^{[4]}$. Sitam et al ${ }^{[5]}$ claimed that bone fracture in postmenopausal osteoporotic women was related to the low level of estrogen which in turn stimulated interleukin-1 (IL-1), interleukin-6 (IL-6), transforming growth factor- $\beta$ (TGF- $\beta$ ) and receptor activator of nuclear factor- $\kappa \mathrm{B}$ ligand (RANKL).

Glucocorticoids (GCs) are extensively utilized for treating autoimmune diseases, rheumatism, gastrointestinal diseases, tumors and organ transplantation for decades. Glucocorticoid-induced osteoporosis (GIO) is one of the significant side effects which have evolved as the most common secondary osteoporosis in adults. It has been noticed that $30 \%$ of patients with long-term (over 6 months) use of GCs acquired osteoporosis. GIO is related to increased osteoblasts apoptosis and decreased osteogenesis ${ }^{[6]}$.

Many approaches have been assessed in preventing and treating GIO among which are the traditional bisphosphonates and teriparatide ${ }^{[7]}$. Since there are many issues regarding their efficacy and side effects, the need for new therapeutic approaches is alleviating. 
Mesenchymal Stem Cells (MSCs) have been used to enhance bone repair and were studied in different animal species $^{[8,9]}$. Furthermore, clinical trials used MSCs for treating bone injuries in oral defects as well as maxillofacial and long-bone defects ${ }^{[10]}$. The mechanisms by which MSCs promote tissue repair include, paracrine activity through secretion of proteins/peptides and hormones; transfer of mitochondria through secretion of microvesicles and transfer of exosomes or microvesicles containing RNA and other molecules ${ }^{[11]}$.

Microvesicles (MVs) have potential therapeutic entities for tissue repair, an established role in intercellular communication and stem cell regulation ${ }^{[12,13]}$. MVs are membrane fragments that effuse from most of the eukaryotic cells during activation or apoptosis. MVs can stash not only lipids from the cell surface and membrane proteins, but also nucleic acids including but not limited to messenger RNAs (mRNAs), microRNAs (miRNAs), small-interfering RNAs (siRNAs) and long non-coding RNAs (lncRNAs) from the intracellular environment. Therefore, as they simulate various components from their parental cells; MVs could perform various functions in intercellular communication, signal transduction and immune regulation ${ }^{[14]}$.

Despite multiple stem cell-based biomaterials and products are presently being investigated, comparative studies are rarely achieved to evaluate the most appropriate approach in this context. Thus, the present study compared between the regenerative capacities of mesenchymal stem cells and microvesicles in accelerating bone regeneration in TMJs of glucocorticoid-induced osteoporotic albino rats.

\section{MATERIALS AND METHODS}

\section{Isolation of bone marrow mesenchymal stem cells and microvesicles}

\subsection{Isolation and identification of bone marrow mesenchymal stem cells (BMMSCs)}

Bone marrow was extirpated out from tibiae of 5 rats (10-weeks-old) using phosphate buffer saline (PBS) (Gibco/Invitrogen, Grand Island, New York, USA) and centrifuged at $1000 \mathrm{rpm}$ for $5 \mathrm{~min}$. The cells were cultured within an RPMI medium (Gibco BRL, USA), 10\% fetal bovine serum (FBS, Gibco BRL, USA) and kept in a cell culture incubator containing $5 \% \mathrm{CO} 2$ at $37^{\circ} \mathrm{C}$. At $80-90 \%$ BMMSCs confluence, they were detached with $0.25 \%$ trypsin-EDTA (Gibco BRL, USA) and subcultured in new flasks. Third passage BMMSCs were used; which were characterized by their morphological spindle shape. Further identification of BMMSCs was established by flow cytometry (Beckman Coulter). They were suspended (1x 106 cells $/ \mathrm{ml}$ ) and stained with FITC conjugated monoclonal antibodies, CD34, CD90 and CD105.

\subsection{Isolation and identification of microvesicles (MVs)}

MVs were obtained from supernatants of third passage BMMSCs $(5 \times 106$ cells $/ \mathrm{ml})$ cultured in RPMI deprived of FBS and supplemented with $0.5 \%$ of bovine serum albumin (BSA) (Sigma). After $20 \mathrm{~min}$ from centrifugation at $2000 \mathrm{~g}$ to remove debris, supernatants free from cells were centrifuged at $100000 \mathrm{~g}$ (Beckman Coulter Optima $\mathrm{L} 90 \mathrm{~K}$ ultracentrifuge) for $1 \mathrm{~h}$ at $4^{\circ} \mathrm{C}$, washed in serumfree medium 199 containing HEPES 25mM (Sigma) and submitted to the second ultracentrifugation under the same conditions. The protein content was quantified by the Bradford method (BioRad, Hercules, CA). Purified MVs were then cultured overnight in a medium used for the collection of MVs.

\subsection{FACS Analysis of MVS}

The MVs were identified by cytofluorimetric analysis using FITC-conjugated antibodies; CD44, CD63 (Miltenyi Biotec) and CD73 (Becton Dickinson). FITC mouse nonimmune isotypic IgG (Dako Cytomation) was used as control.

\section{Experimental Procedures}

The study was conducted on thirty-nine adult female albino rats with an average weight of 150-200 gm. The rats were housed singly in specially designed cages. They were kept under good ventilation and had a free approach to both water and standard rodent soft chow ad-libitum. All the experimental procedures were performed in the animal house of the Faculty of Medicine, Cairo University, according to the recommendations and approval of the Institutional Animal Care and Use Committee (IACUC), Cairo University (CU/III/F1719).

\subsection{Induction of osteoporosis and animal grouping}

All the rats received an intraperitoneal injection once daily throughout the experimental period (60 days); 13 rats received $9 \%$ saline while the remaining 26 rats received dexamethasone $(200 \mu \mathrm{g} / 100 \mathrm{~g}$ body weight $)$ for induction of osteoporosis ${ }^{[15]}$. After 30 days; the first group (control group) received a local injection of $1 \mathrm{ml}$ PBS in TMJ of both sides. Half of the dexamethasone injected rats received a local injection in the right side TMJ of $1 \times 107$ $/ \mathrm{ml} /$ week MVs in PBS (Microvesicles group). The other half (Stem cells group) received 1 million MSCs/ ml/week. In the later 2 groups, the left side TMJs received a local injection of $1 \mathrm{ml}$ PBS.

After 30 days from induction of osteoporosis, BMD of the alveolar bone was measured at the molar region for 5 random samples from each of the control and the osteoporosis groups using Norland XR-46 DEXA scanner (Norland Corp. Fort Atkinson, WI, USA) equipped with appropriate software for bone assessment in small animals 
in the Egyptian National Research Institute. The scan resolution was $0.5 \times 0.5 \mathrm{~mm}$ and the scan speed was 60 $\mathrm{mm} / \mathrm{sec}$. Results were displayed in $\mathrm{gm} / \mathrm{cm} 2$ (Mean $\pm \mathrm{SD}$ ).

At the end of the experiment period (60 days); all rats were sacrificed with ketamine overdose. The heads were sagittally dissected, fixed in $10 \%$ neutral formalin for 48 h. The samples were used in histological examination and histomorphometric analysis as well as for western blotting for RANKL expression, ELISA for IL- $1 \beta$, TNF- $\alpha$, IL-6 and RT-PCR for ALP, BMP and RUNX-2. The results of both sides of the control group were averaged and used as a single value for each sample.

\section{Light microscopic examination}

Specimens from each experimental group were washed and soaked in $10 \%$ ethylene diamine tetra-acetic acid (EDTA) for 4-5 weeks for decalcification. The specimens were dehydrated in ascending grades of alcohol following decalcification, then cleared in xylol and embedded in paraffin blocks. Sections of 4-5 $\mu$ thickness were mounted on ordinary glass slides and stained by Hematoxylin and Eosin (H\&E) for routine histological evaluation according to the conventional method.

\section{Histomorphometric analysis}

In the hematoxylin and eosin-stained sections, the bone area percentage was measured using Leica Qwin 500 image analyzer computer system (England). The measurements were taken using an objective lens of magnification 10 , that is, of a total magnification of 100 . Ten fields were measured from each sample and the mean values were calculated.

\section{Western blotting}

The antibody used was antigen affinity-purified receptor activator of nuclear factor- $\kappa B$ ligand (RANKL) monoclonal antibody (Santa Cruz-52950). The Ready Prep $^{\mathrm{TM}}$ protein extraction kit (Cat \#163-2086, BioRad inc., CA, USA) was applied for protein extraction procedure from the tissues. Bradford protein assay kit (Cat \#SK3041, Bio basic inc., Ontario, Canada) was employed for quantitative protein analysis. Protein samples were separated on a polyacrylamide gel (TGX Stain-Free Fast Cast Acrylamide Kit). Then $20 \mu \mathrm{g}$ of total protein was loaded per each mini-gel well. The gel was then gathered in the transfer sandwich with a polyvinylidene difluoride (PVDF) membrane. Protein bands were allowed to transfer from gel to membrane using BioRad Trans-Blot Turbo. PVDF blot was incubated in 5\% non-fat dry milk, TrisHCL, $0.1 \%$ Tween 20 for $1 \mathrm{hr}$. RANKL antibody was added to a membrane containing specimen samples and incubated at $4{ }^{\circ} \mathrm{C}$ overnight. The appropriate secondary antibody was incubated for $2 \mathrm{hr}$ at room temperature. After being washed twice in $1 \mathrm{x}$ TBS-T, densitometric analysis of the immunoblot was performed to quantify the amounts of RANKL in all studied samples against control sample beta-actin (housekeeping protein) by protein normalization on the Chemi Doc MP imaging system (version 3) manufactured by Bio-Rad (Hercules, CA). The Western blotting procedures had been performed in the Biochemistry Department, Faculty of Medicine, Cairo University.

\section{ELISA and qRT-PCR}

The ELISA and qRT-PCR procedures had been performed in the Biochemistry Department, Faculty of Medicine, Cairo University.

\subsection{Samples processing}

One gram was weighted from the tissue. Tissue was homogenized (Con-Torque Eberbach's Tissue Homogenizer, Michigan) in $1 \mathrm{ml}$ PBS. Each homogenate was centrifuged at $10000 \mathrm{rpm}$ speed and $5^{\circ} \mathrm{C}$ temperature for 10 minutes using cooling centrifuge (Sigma Aldrich). Each supernatant was collected into a new microcentrifuge tube $1.5 \mathrm{ml}$. The collected supernatant was used for the following.

\subsection{ELISA}

Tumor necrosis- $\alpha$ (TNF- $\alpha$ ) level (pg/g tissue) (Sigma Aldrich, Catalog Number: RAB0480), IL-6 level (pg/g tissue) (RayBio ${ }^{\circ}$, Catalog \#: ELR-IL6) and IL-1 $\beta$ level (pg/g tissue) (RayBio ${ }^{\circ}$, Catalog \#: ELR-IL1 $\beta$ ) were assessed in tissue homogenate according to manual instructions.

\subsection{RNA Extraction}

Tissues from all studied groups were lysed and total RNA was extracted using RNAeasy Mini Kit (Qiagen) and further analyzed for quantity and quality with Beckman dual spectrophotometer (USA).

\section{4. $q R T-P C R$}

For the quantitative expression of alkaline phosphatase (ALP), bone morphogenetic protein (BMP) and runt-related transcription factor-2 (RUNX-2); the following procedures were performed. From each sample; $10 \mu \mathrm{g}$ from the total RNA was used for cDNA synthesis by reverse transcription using a high capacity cDNA Reverse Transcriptase kit (Applied Biosystem, USA). The cDNA was amplified with the Syber Green I PCR Master Kit (Fermentas) in a 48-well plate using the Step One instrument (Applied Biosystem, USA). Amplification involved; 10 minutes at $95{ }^{\circ} \mathrm{C}$ for enzyme activation; 40 cycles of 15 seconds at $95^{\circ} \mathrm{C} ; 20$ seconds at $55^{\circ} \mathrm{C}$ and finally 30 seconds at $72{ }^{\circ} \mathrm{C}$. Changes in the expression of each target gene were normalized relative to the mean critical threshold (CT) values of GAPDH as housekeeping gene by the $\Delta \Delta \mathrm{Ct}$ method. $1 \mu \mathrm{M}$ of both primers specific for each target gene was used. Primers sequence specific for each gene demonstrated in (Table 1). 
Table 1: Primers sequence specific for each gene

\begin{tabular}{|c|c|c|}
\hline Target gene & Primer sequence: $5^{`}-3^{`}$ & Gene bank accession number \\
\hline ALP & $\begin{array}{l}\text { F: ACGTGGCTAAGAATGTCATC } \\
\text { R: CTGGTAGGCGATGTCCTTA }\end{array}$ & NM000660.2 \\
\hline BMP & $\begin{array}{l}\text { F: GCGAGGTGGTACTTGGTCTG } \\
\text { R: GAGAGGGTGAGGCTCTGTTG }\end{array}$ & NM198253.2 \\
\hline RUNNX-2 & $\begin{array}{l}\text { F: GTTATGAAAAACCAAGTAGCCAGGT } \\
\text { R: GTAATCTGACTCTGTCCTTGTGGAT }\end{array}$ & NM009820 \\
\hline GAPDH & $\begin{array}{l}\text { F : A C A G T C C A T G C C A T C A C T G C C } \\
\text { R:GCCTGCTTCACCACCTTCTTG }\end{array}$ & NG009348.3 \\
\hline
\end{tabular}

\section{Statistical analysis}

Values were presented as mean and standard deviation (SD). Kolmogorov-Smirnov test of normality was used to explore data normality. The results of the KolmogorovSmirnov test indicated that most of the data were normally distributed (parametric data), therefore, one-way analysis of variance (ANOVA) test was used between groups. This was followed by Tukey's post hoc test when ANOVA yielded a significant difference. The significance level was set at $p<0.05$. Statistical analysis was performed with SPSS 18.0 (Statistical Package for Scientific Studies, SPSS, Inc., Chicago, IL, USA) for Windows.

\section{RESULTS}

\section{Induction of osteoporosis and DEXA results}

BMD of the alveolar bone at the molar region revealed a significant decrease $(P<0.001)$ in rats that were injected with dexamethasone $(0.08938 \pm 0.007005)$ as compared to control rats $(0.1398 \pm 0.001716)$.

\section{Characterization of MSCs and MVs}

Isolated MSCs were identified using inverted microscope by their morphology (fibroblast like cells) (Figure 1A). MVs were analyzed by electron microscopy and identified as spheroids with average diameter $75.5 \mathrm{~nm}$ (Figure 1B). FACS analysis were done for confirmation of isolated cells and showed CD90+, CD105+ and CD34for MSCs (Figure 1C) and CD44+, CD63+ and CD73+for MVs (Figure 1D).

\section{Histological results}

\subsection{Control group}

The rat's TMJ consisted of mandibular condyle, articular disc and temporal bone. The head of the condyle was composed of spongy bone. The bony trabeculae appeared as interconnecting plates lined by osteoblasts along their borders and enclosing marrow spaces. Numerous regularly arranged osteocytes could be distinguished entrapped within their lacunae. Moreover, resting lines were detected. The articulating surface of the condylar head was covered with a relatively thick layer of fibrocartilagenous tissue which was further underlined by a well-defined layer of hyaline cartilage with its characteristic oval-shaped chondrocytes. Regularly arranged fibroblasts oriented parallel to each other and the joint cavity could be observed together with few dispersed chondrocytes. A biconcave disc; with its central area thinner than its margins was also apparent in the specimens. It was made up of fibrous connective tissue and divided the joint cavity into two compartments; a large superior and a smaller inferior one. Similar to the condylar head, the temporal bone was composed of interconnected plates of spongy bone; some of which assumed the concentric configuration and its articulating surface was covered by fibrocartilage (Figure 2).

\subsection{Microvesicles group}

Histological findings of the examined specimens of both sides revealed nearly normal configuration of the TMJ. Yet, widening of the marrow spaces was still evident in the joints of the left side (untreated) particularly within the temporal bone (Figure 3B) when compared to the opposing side (treated) (Figure 3A). On higher magnification, the bone trabeculae in the untreated side assumed irregular patterns with haphazardly entrapped osteocytes. The osteoblasts partially bordered the trabecular boundaries which enclosed marrow spaces with numerous cells and extravasated RBCs (Figure 3D). Regarding the joints of the right side (treated), the condylar head with its regular trabecular pattern enclosing osteocytes was apparent together with a well-developed layer of hyaline cartilage and an overlying fibrocellular layer covering the articular surface facing the joint cavity. The radiating bone trabeculae displayed a more uniform configuration with regularly arranged osteocytic cells (Figures 3A,C). Moreover, the marrow spaces of the treated side expressed numerous, deeply stained cells that began to differentiate and become arranged along the borders of the bone resembling osteoblasts. Some of these cells exhibited a spindle configuration; others assumed a giant cell appearance (Figure 3C). The temporal surface of the untreated side appeared with widened and interconnected marrow spaces (Figure 3B). On the contrary, the temporal surface of the treated side had smaller marrow spaces enclosing numerous cellular elements (Figure 3A).

\subsection{Stem Cells Group}

Unlike the microvesicles group, an abnormal configuration of the joint's anatomy was revealed on histological examination of both sides whether treated or untreated. Disfigured condylar head with the absence of 
the normal trabecular pattern of spongy bone together was noticed. The disappearance of the characteristic hyaline cartilage layer and the regular fibrocartilagenous covering was further noticed (Figure 4).

Regarding the right side joints (treated) (Figure 4A); the head of the condyle appeared to be composed of lamellar bone with different orientations and arrangements. At the joint cavity (articular surface), the lamellae were arranged parallel to the surface; whereas the bulk of the head exhibited thickened closely packed lamellae enclosing osteocytes. Minute spaces were detected simulating marrow spaces (Figure 4A) which were partially outlined by osteoblastic cells (Figure 4C). On the other hand, the marrow spaces were extremely widened and interconnected in the condylar head of the TMJ specimens of the left side (untreated) (Figure 4B). Furthermore, they were fully occupied by highly cellular and vascular tissue. Thin branching bone trabeculae with a fewer entrapped population of osteocytes in widened lacunae were detected in the left side joint. Sometimes, the lacunae were empty. Osteoblasts disappeared in focal dispersed areas in the untreated side (Figure 4D). The temporal bone in both sides appeared to be formed of interconnected trabeculae with intervening marrow spaces enclosing cellular elements. Those spaces were widened in the untreated than the treated side (Figures 4A, B). Nevertheless, the joints of both sides displayed apparent thickening of the fibrous lining of the articular surface.

\section{Histomorphometric analyses}

A significant difference in the bone area \% between all the studied groups has been recorded $(p<0.001)$. A significant decrease in bone area percentage was observed in the TMJs of both sides of the stem cells group (either treated or untreated) as compared to the control group $(p<0.001)$, while no significant difference between both sides of the microvesicles group (either treated or untreated) compared to the control group ( $p=0.61$ and 0.98 , respectively) was recorded. A significant increase in bone area $\%$ was detected in untreated sides of MVs group as compared to the untreated MSCs group $(p<0.001)$. Moreover, a significant decrease in bone area percentage has been recorded in the untreated TMJs of stem cells group as compared to the treated ones $(p=0.044)$. On the other hand, a non-significant difference in bone area percentage between both sides of the MVs group has been reported $(\mathrm{p}=0.893)$ (Figure 5).

\section{5. $R A N K L$ protein expression}

A significant difference has been recorded between all groups regarding RANKL expression $(p<0.001)$. RANKL protein expression was significantly higher in untreated MSCs, MVs groups and treated MVs group as compared to control group ( $p<0.001,<0.001$ and 0.03 , respectively), while no significant difference between treated MSCs and control groups $(\mathrm{p}=0.99)$ was recorded. Furthermore, no significant difference was revealed between untreated MSCs and untreated MVs groups ( $p=0.61$ ), yet there was a significant decrease in RANKL expression in treated side compared to the corresponding untreated side in both MSCs and MVs groups $(p<0.001)$. No significant difference was evident between untreated MSCs and untreated MVs groups $(\mathrm{p}=0.054)$ (Figure 6).

\section{ELISA results for the inflammatory markers}

The inflammatory cytokines (IL-1 $\beta$, IL- 6 and TNF- $\alpha$ ) were significantly higher in untreated MSCs, untreated MVs groups and treated MVs group as compared to control group $(p<0.001)$ while no significant difference existed between treated MSCs and control group $(\mathrm{p}=0.9)$. For TNF- $\alpha$, no significant difference between untreated MSCs and untreated MVs groups was revealed $(\mathrm{p}=0.12)$, while there was a significant decrease in IL-1 $\beta$ and IL-6 levels in untreated MVs compared to untreated MSCs $(p<0.001)$. For all cytokines, a significant decrease in their levels in treated side as compared to the corresponding untreated side in both MSCs and MVs groups $(p<0.001)$ was evident. Also a significant decrease in their levels in treated MSCs group as compared to treated MVs group was found $(p<0.001)$. (Figures 7 A, B, C)

\section{7. qRT-PCR results for the osteogenic markers}

The osteogenic markers (ALP, BMP and RUNX-2) expressions were significantly decreased in untreated MSCs, untreated MVs groups and treated MVs group as compared to control group $(p<0.001)$ while there was a significant increase in ALP and BMP genes expression in treated MSCs as compared to the control group $(p<0.001)$. A non significant difference in RUNX-2 gene expression was detected between treated MSCs and control group $(p=0.79)$. For BMP and RUNX-2, no significant difference existed between untreated MSCs and untreated MVs groups ( $\mathrm{p}=0.48$ and 0.24 , respectively), while a significant increase in ALP expression in untreated MVs as compared to untreated MSCs ( $p=0.015)$. For all markers, a significant increase in their expressions was recorded in treated sides as compared to the corresponding untreated sides in both MSCs and MVs groups $(p<0.001)$. Moreover, a significant increase in their expression in treated MSCs group as compared to treated MVs group was apparent $(p<0.001)$. (Figures 7 D, E, F) 


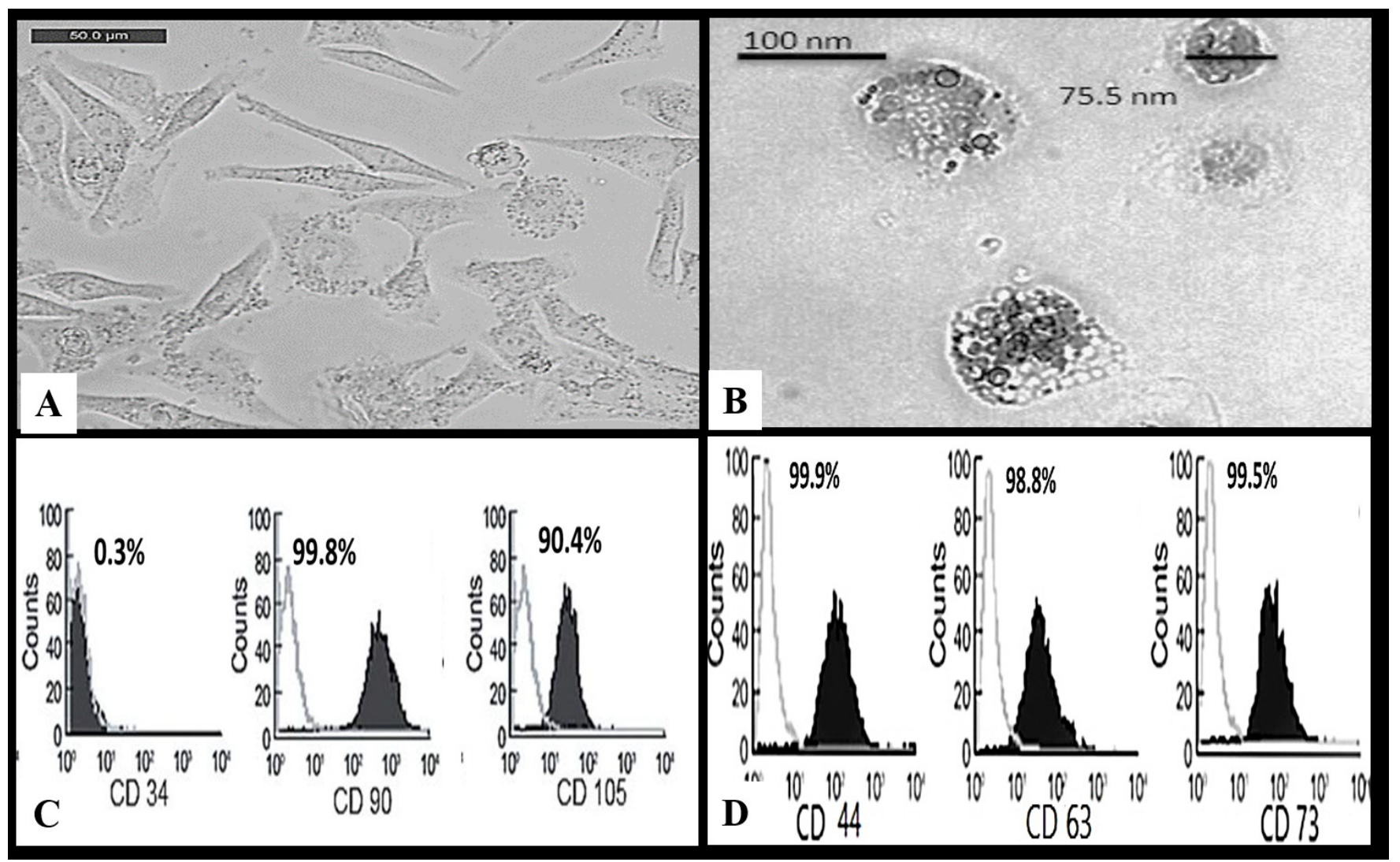

Fig. 1: (A) showing MSCs having fibroblast like shaped under inverted microscope. (B) showing MVs scanned with electron microscope with a spheroid shape 75.5nm. (C) FACS analysis showing CD90+, CD105+ and CD34- for MSCs. (D) FACS analysis showing CD44+, CD63+ and CD73+for MVs.

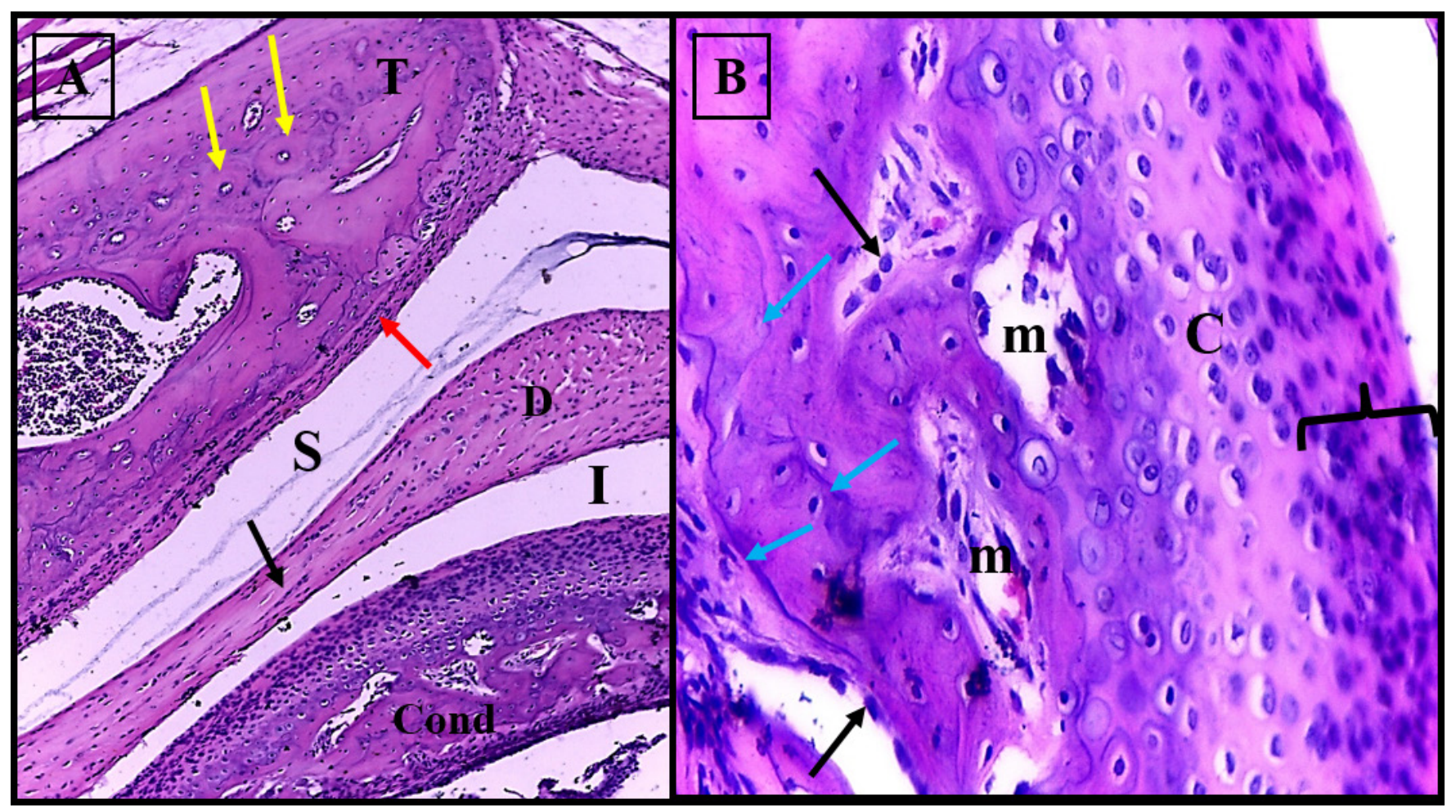

Fig. 2: A photomicrograph of TMJ of control group showing;

(A) Mandibular condyle (Cond), biconcave articular disc (D) with thin central area (black arrow) dividing the joint cavity into superior (S) and inferior (I) compartments and temporal bone (T) composed of interconnected plates of spongy bone some of which assumed concentric configuration (yellow arrows) with its articulating surface covered by fibrocartilagenous tissue (red arrow) (H\&E, Orig. Mag. X4)

(B) Higher magnification of the condylar head composed of spongy bone with interconnecting bone trabeculae lined by osteoblasts along their borders (black arrows), enclosing marrow spaces (m), resting lines (blue arrows), covered with a relatively thick layer of fibrocartilagenous tissue with parallel oriented fibroblasts (bracket) and underlined by a layer of hyaline cartilage (C) (H\&E, Orig. Mag. X40) 


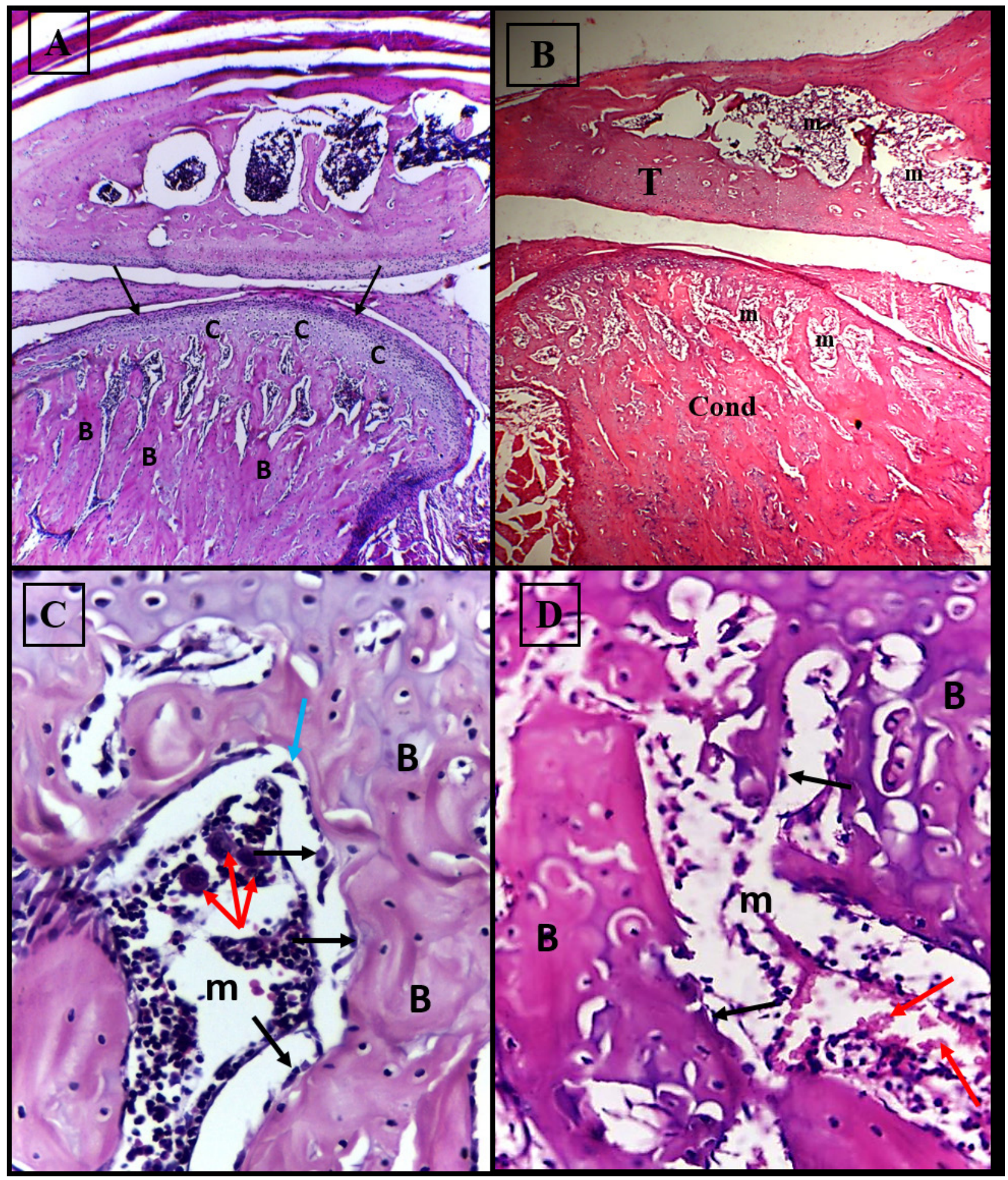

Fig. 3: A photomicrograph of TMJ of microvesicles group A, C (treated side) and B, D (untreated side) showing;

(A): Nearly normal configuration of the TMJ with radiating bone trabeculae (B), a well developed layer of hyaline cartilage (C) and an overlying fibrocellular layer covering the articular surface (arrows) (H\&E, Orig. Mag. X 4)

(B): Widening of the marrow spaces (m) within the condyle (Cond) and temporal bone (T) (H\&E, Orig. Mag. X 4)

(C): Higher magnification showing; bone trabeculae (B) with regularly arranged osteocytes, marrow spaces (m) expressing numerous, deeply stained differentiating cells which apparently began to be arranged along the borders of the bone resembling osteoblasts (black arrows), some of these cells exhibited a spindle configuration resembling UMCs (blue arrows); others assumed a giant cell appearance (red arrows) (H\&E, Orig. Mag. X 40)

(D): Higher magnification showing; irregular pattern of bone trabeculae (B) with haphazardly entrapped osteocytes, osteoblasts partially bordering the trabecular boundaries (black arrows), marrow spaces (m) with numerous cells and extravasated RBCs (red arrows) (H\&E, Orig. Mag. X40) 


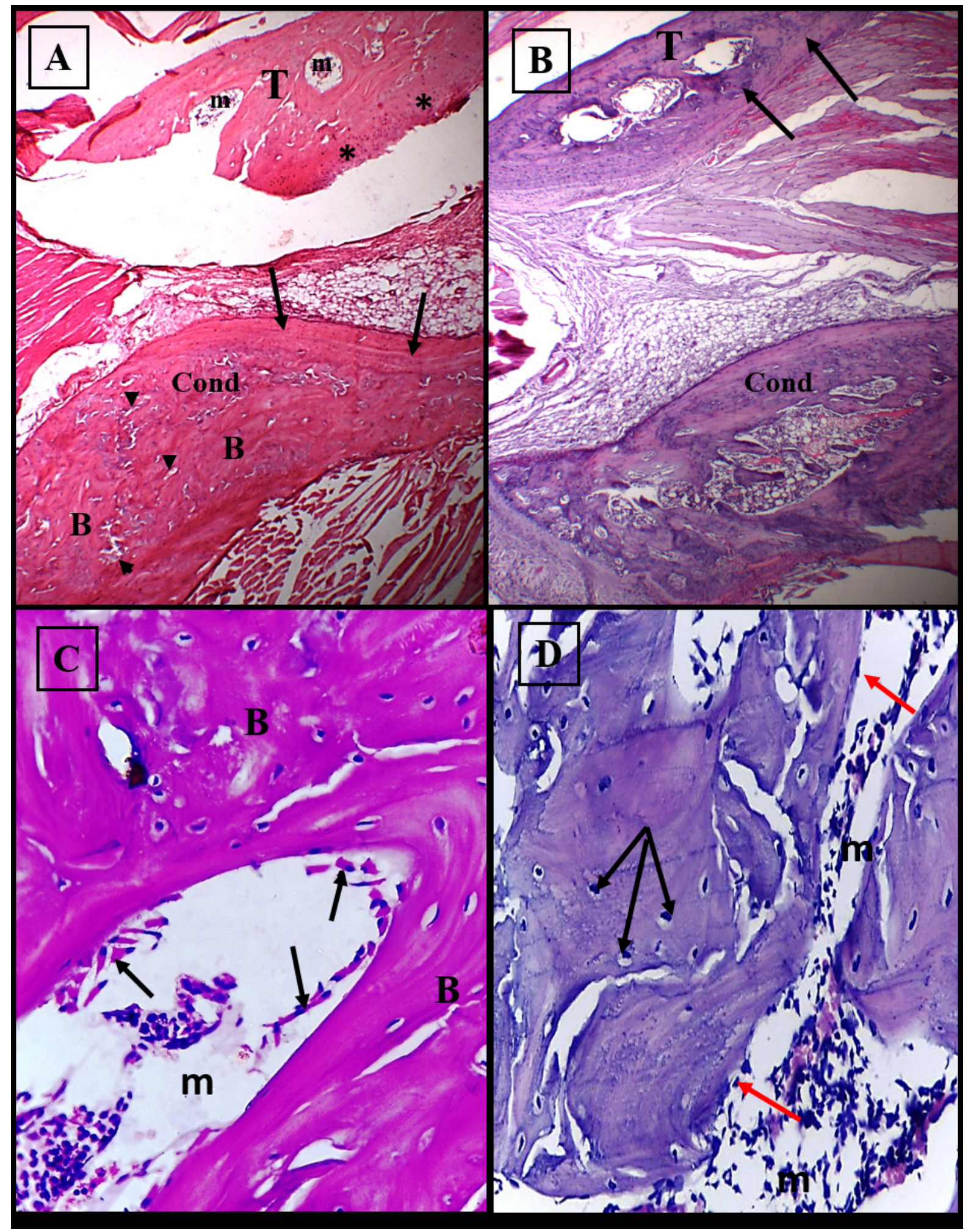

Fig. 4: A photomicrograph of TMJ of stem cells group A, C (treated side) and B, D (untreated side) showing;

(A): Abnormal configuration of the joint's anatomy where the head of the condyle (Cond) appeared to be composed of lamellar bone with different orientation and arrangement; parallel lamellae at the joint cavity (articular surface) (arrows); thickened bone trabeculae (B) at the bulk of the condyle; minute spaces simulating marrow spaces (arrowheads); temporal bone $(\mathrm{T})$ formed of interconnected trabeculae with intervening marrow spaces (m) and thickened fibrous lining (asterisks) (H\&E, Orig. Mag. X 4)

(B): Disfigured condylar head (Cond) with extremely widened and interconnected marrow spaces, temporal bone (T) with widened intervening marrow spaces enclosing cellular elements with apparent thickening of the fibrous lining of the articular surface (arrows) (H\&E, Orig. Mag. X 4)

(C): Higher magnification showing; bone trabeculae (B) with numerous entrapped osteocytes; marrow spaces (m) partially outlined by osteoblastic cells and UMCs (arrows) (H\&E, Orig. Mag. X 40)

(D): Higher magnification showing; few entrapped population of osteocytes (black arrows); in some areas, osteoblasts outlining the boundaries of bone (red arrows) and marrow spaces (m) fully occupied by highly cellular and vascular tissue (H\&E, Orig. Mag. X 40) 


\section{Bone area \%}

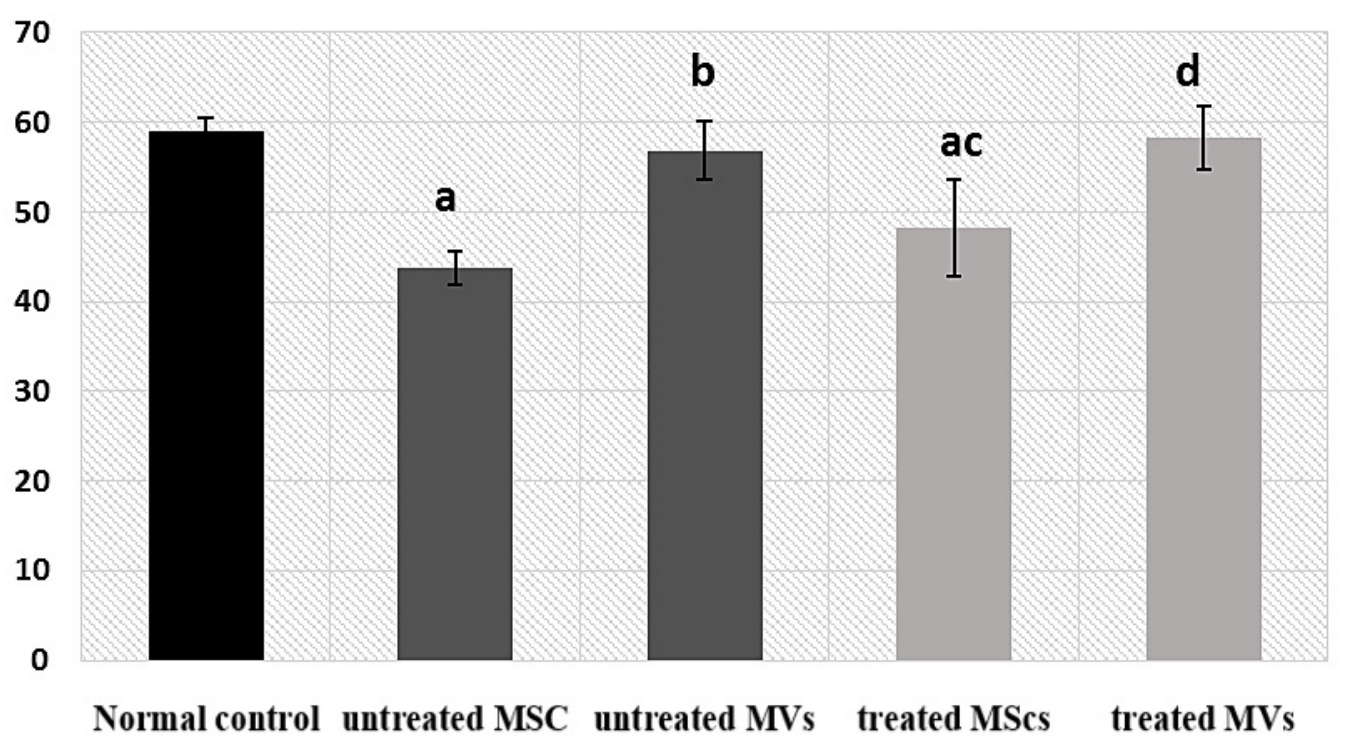

Fig. 5: Showing bone area \% - Data were expressed as mean \pm SD. $p$ value $<0.05$ is considered significant. (a) Denotes significant difference versus control group. (b) Denotes significant difference between untreated MSCs and untreated MVs groups (c) Denotes significant difference versus corresponding untreated group (d) Denotes significant difference between treated MSCs and treated MVs groups

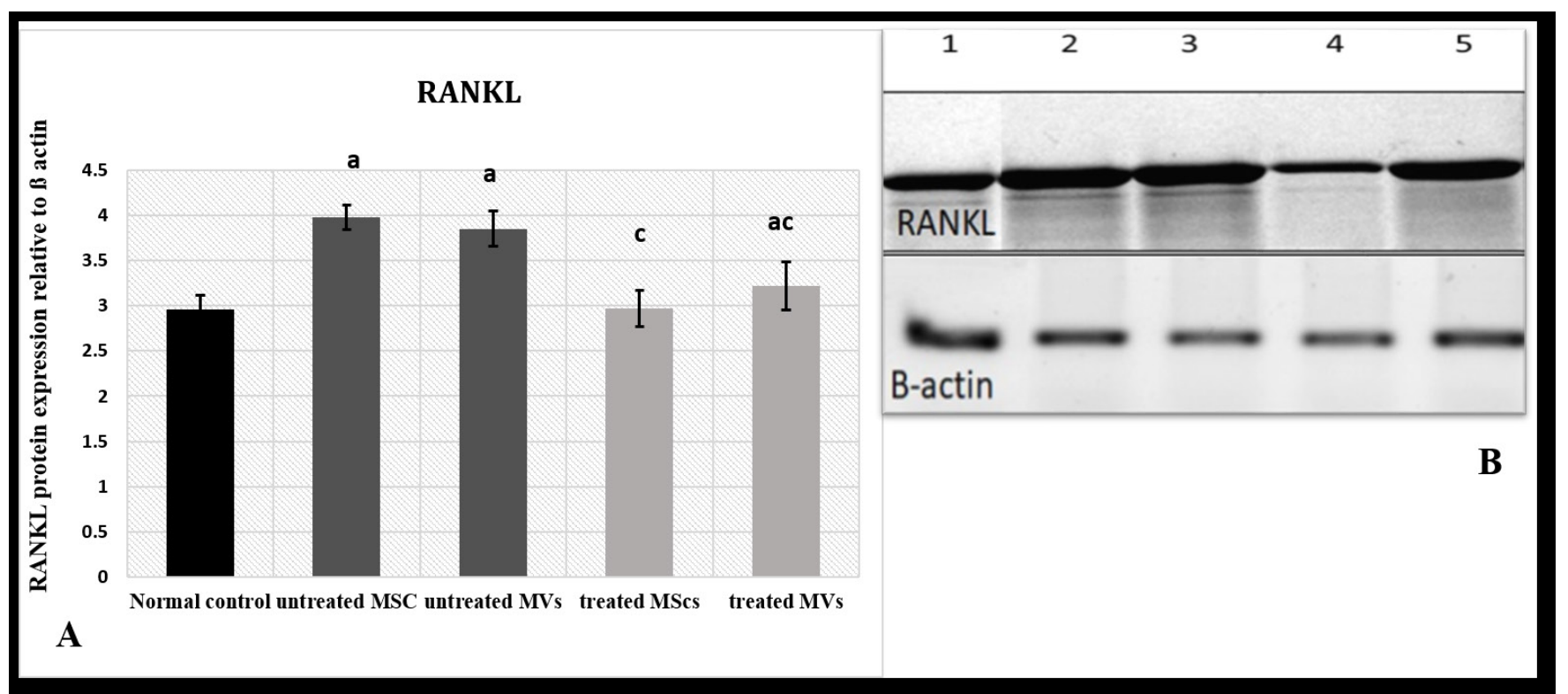

Fig. 6: Showing RANKL protein expression by western blotting technique. Data were expressed as mean \pm SD. $\mathrm{p}$ value $<0.05$ is considered significant. (a) Denotes significant difference versus control group. (c) Denotes significant difference versus corresponding untreated group 


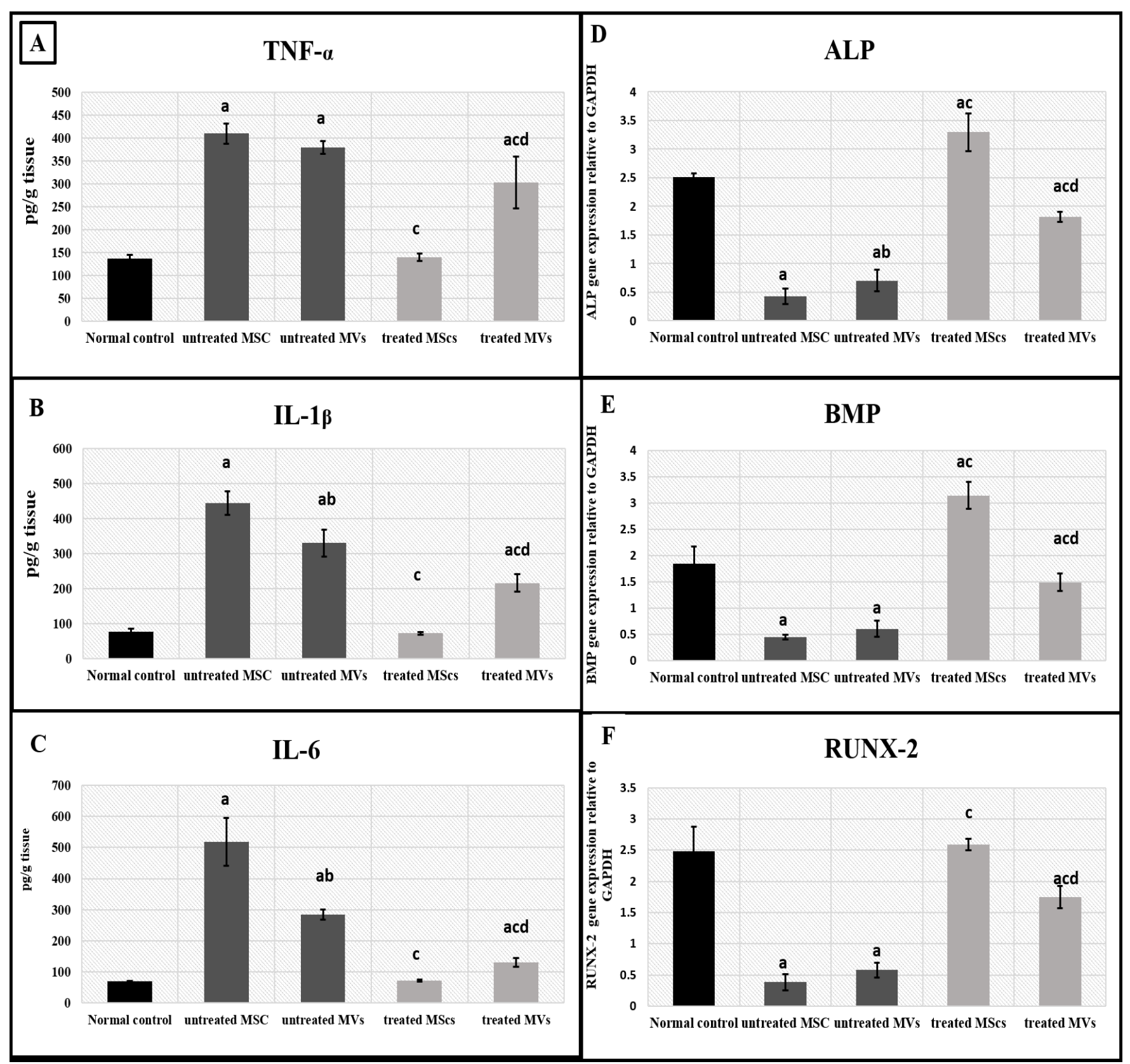

Fig. 7: Showing inflammatory cytokines levels by ELISA technique; A: TNF- $\alpha$, B:IL-1 $\beta$, C: IL-6. as well as osteogenic bone markers expression by RT-PCR technique; D: ALP, E: BMP, F: RUNX-2)

Data were expressed as mean $\pm \mathrm{SD}$. $p$ value $<0.05$ is considered significant

(a) Denotes significant difference versus control group.

(b) Denotes significant difference between untreated MSCs and untreated MVs groups

(c) Denotes significant difference versus corresponding untreated group

(d) Denotes significant difference between treated MSCs and treated MVs groups 


\section{DISCUSSION}

Osteoporosis is considered a major complication of corticosteroid therapy ${ }^{[16]}$. Glucocorticoid-induced osteoporosis (GIO) is associated with decreased osteogenesis and increased osteoblastic and osteocytic apoptosis that consequently result in reduced bone formation and decreased $\mathrm{BMD}^{[17]}$. Treating osteoporosis with drugs such as bisphosphonates, causes adverse effects, including osteonecrosis of the jaws, atypical femur fractures, atrial fibrillation and esophageal cancer ${ }^{[18]}$. Consequently, the need for new approaches for treating osteoporosis is alleviating.

The ongoing study aimed to investigate the bone regenerative capacity of MVs as compared to MSCs in treating the histopathological changes associated with GIO in rats' TMJ. TMJs bony components are covered by fibrocartilagenous tissue; that makes the bone more reliable to inflammatory deterioration consequent to systemic disease and a worthy model for studying bony changes ${ }^{[19]}$.

For ethical reasons, an appropriate animal model minimizes the limitations associated with studying the disease in humans ${ }^{[20]}$. Morphologically and histologically, the structure of rats' TMJ is, on the whole, similar to that of humans ${ }^{[21]}$. Since the incidence of osteoporosis is more in women, the ovariectomized rats is considered the most common model used in research for postmenopausal osteoporosis. Moreover, the glucocorticoid-induced osteoporosis is an alternative method to surgical ovariectomy ${ }^{[22]}$, thus, in the present study, osteoporosis was induced in female rats via glucocorticoids.

The induced bone loss was confirmed after 4 weeks by a significant decrease in BMD in the alveolar bone. Accordingly, Weinstein ${ }^{[23]}$ reported that mice receiving GCs for 4 weeks (a period equivalent to about 3 to 4 years in humans) exhibited an early rapid and later slow loss of BMD. This loss was associated with a reduction in cancellous bone, trabecular width and rate of bone formation together with a decrease in the number of osteoblastic and osteoclastic progenitor cells in the bone marrow.

In the ongoing study following the administration of dexamethasone for 60 days, the head of the condyle of the untreated TMJs from the MSCs group revealed widened marrow spaces and thin branching trabeculae with dispersed osteocytes in widened lacunae. Osteoblasts could be seen partially outlining their boundaries rather than completely encircling them. Moreover, the temporal surface appeared to be composed of spongy bone with extremely widened marrow spaces. Concomitantly, Ezzat $\&$ Abbass $^{[15]}$ observed marked alterations in the spongiosa of glucocorticoid group in the form of wide marrow spaces surrounded with multiple reversal lines and shrunken osteocytes in wide lacunae. The widened osteocytic lacunae observed in this group in the current work could be due to local degradation; a phenomenon known as osteocytic osteolysis. This phenomenon was observed and explained by Lane et al. ${ }^{[24]}$ who confirmed that glucocorticoids induce osteocytes apoptosis; as well as cause enlargement of the osteocytes lacunae. Osteocytes remove minerals from the perilacunar space thereby generating "halos" of hypomineralized bone.

GCs, particularly at high concentrations, tend to reduce the number of osteoblasts and osteocytes by apoptosis due to caspase -3 activation ${ }^{[25]}$. Moreover, GCs inhibit the synthesis of type I collagen by osteoblasts, with a subsequent decrease in bone matrix essential for mineralization ${ }^{[26]}$. Furthermore, GCs increase the expression of RANKL; which is produced by osteocytes and control osteoclastogenesis and decrease the expression of osteoprotegerin (OPG) that physiologically counterbalance the effect of RANKL in osteoblasts ${ }^{[27]}$. GCs also enhance the expression of IL-6, an osteoclastogenic cytokine and suppress the expression of interferon-beta, an inhibitor of osteoclastogenesis $^{[28]}$. Recently, it has been reported that the apoptotic bodies released from osteocytes due to GCs treatment might enhance the production of TNF- $\alpha$ and IL6 . Furthermore, TNF- $\alpha$ can induce osteocyte apoptosis and contribute to a worsened bone quality ${ }^{[29]}$.

In the current study, the histological and biological osteoporotic changes were shown apparently in the untreated TMJs from the MSCs group; where the expression of RANKL and other inflammatory markers were significantly increased as compared to the control group as well as to both sides of the microvesicles group. While the osteogenic markers and the bone area \% were significantly decreased in this group as compared to the former groups. Accordingly, Jabbar et al. ${ }^{[30]}$ deduced that the circulating levels of OPG and RANKL were inversely related to BMD and contributed to the development of osteoporosis in postmenopausal women.

In the present study, histological examination of both sides (treated and untreated) MSCs group, revealed abnormal configuration of the joint's anatomy. Disfigured condylar head with the absence of the normal trabecular pattern of spongy bone. The reported deformity disagrees with Kiernan et al. ${ }^{[31]}$ who mentioned that the systemic administration of allogeneic MSCs in an age-related osteoporotic mouse, markedly improved bone formation and maintained bone architecture. In the current work, the reported loss of normal TMJ architecture in the treated MSCs sides despite the significant increase in all osteogenic markers (RUNX-2, BMP and ALP) and the significant decrease in RANKL expression could be attributed to the excessive bone formation with severe narrowing of the marrow cavities that subsequently decreased the blood supply. On the other hand, the loss of architecture in the untreated TMJs from the MSCs group could be referred to the severe histopathalogical changes that occurred as a result of osteoporosis.

Comparing the treated and untreated sides of the MSCs group revealed a significant increase in bone area $\%$ and osteogenic markers (ALP, BMP and RUNX-2) as well 
as a significant decrease in RANKL and all investigated inflammatory markers $(p<0.01)$ in treated sides than in untreated ones. In accordance with these results, Ocarino et al. ${ }^{[32]}$ reported that intra-bone marrow injection of BMMSCs in ovariectomy (OVX) -induced osteoporosis rat model caused improvement in the femur bone mass, stimulated osteoblastogenesis and bone formation. Moreover, transplantation of adipose-derived MSCs was found to reduce bone loss in OVX mice. Interestingly, the migration of the transplanted cells into the bone tissue resulted in a significant decrease of the serum IL- 6 and IL- $1 \beta$ cytokines and a reduction in the gene expression of RANK in the bone ${ }^{[33]}$.

Regarding the osteoporotic rats treated with local injection of microvesicles in the current study; marked improvement of bone architecture was detected either in the treated or untreated sides. The joint assumed the normal configuration of the condyle, disc and temporal bone. A significant increase in the bone area \% has been revealed between both sides of the MVs group and their corresponding sides of the MSCs group. Yet, nonsignificant differences have been reported between TMJs whether treated or untreated by MVs as compared to the control group as well as between each other.

The regenerative potential of MVs was proved by Zhang et al. ${ }^{[34]}$ who demonstrated that the injection of MVs released from human umbilical cord-MSCs could significantly improve blood flow in a rat model of hindlimb ischemia. Moreover, Zhang et al. ${ }^{[35]}$ provided evidence that the exosomes- $\beta$-tricalcium phosphate combined scaffolds could enhance bone repair and regeneration in a rat model of calvarial bone defects. Furthermore, let-7; a miRNA family, was found markedly to promote in vitro the osteogenetic and to suppress the adipogenic capacities of $\mathrm{MSCs}^{[36]}$. Recently, long noncoding RNA was found to regulate osteogenesis of MSCs via the transcriptional activity of NF- $\kappa^{[37]}$.

Comparing MVs treated and untreated-TMJs, a significant increase in the osteogenic markers and a significant decrease in the inflammatory markers, as well as RANKL expression were revealed in the treated sides. Moreover, similar results were reported when comparing the treated MVs - TMJs with the untreated MSCs ones. This could be attributed to the fact that extracellular miRNAs located within MVs circulating in the bloodstream or other body fluids can also be transported horizontally among different cells $^{[38]}$. The interaction takes place through binding of the donor cell miRNA molecules to complementary sequences in the recipient cell mRNAs; inhibiting their translation or promoting their degradation. Thus, miRNAs can influence a range of biochemical processes; including proliferation, apoptosis and regulation of metabolism ${ }^{[39]}$.

\section{CONCLUSION}

A key point in the current research was that the local injection of microvesicles improved the histopathological and biological changes associated with osteoporosis in the treated side and in the opposite side that didn't receive MVs. This could be referred to the rapid action of MVs; as MVs are formed of genetic material, ready to induce its action and easily to transfer from one site to another. On the other hand, the effect of MSCs was markedly localized. This was confirmed by the marked improvement in all the osteogenic markers in the treated side as compared to the untreated side. Nevertheless, if the study was propagated for a longer time interval, MSCs could produce its effect on contralateral side through its paracrine effect. Yet, MSCs injection produced a more localized antiinflammatory effect and a high osteogenic potential than MVs that unfortunately caused a deformity in the TMJ with shrinkage in its size due to excessive bone formation on the expense of the marrow spaces. Thus, MVs could induce a satisfactory, rapid and contralateral regenerative effect while MSCs produce a localized, more potent antiinflammatory effect and over bone formation that might lead to TMJ deformity.

\section{CONFLICTS OF INTEREST}

There are no conflicts of interest

\section{REFERENCES}

1. Lorentzon $\mathrm{M}, \quad$ Cummings $\mathrm{SR}$ Osteoporosis: the evolution of a diagnosis. J Intern Med. 2015 Jun;277(6):650-661 https://doi.org/10.1111/joim.12369

2. Cosman F, de Beur SJ, LeBoff MS, et al. Clinician's guide to prevention and treatment of osteoporosis. Osteoporos Int. 2014 Oct 1;25(10):2359-2381. https://doi.org/10.1007/s00198-014-2794-2

3. Marya CM, Dhingra C. Effect of Osteoporosis on Oral Health. Arch Med. 2015, 8:2 http://www. archivesofmedicine.com/medicine/effect-ofosteoporosis-on-oral health.php?aid $=8455$

4. Bandela V, Munagapati B, Karnati RK, Venkata GR, Nidudhur SR. Osteoporosis: its prosthodontic considerations-a review. J Clin Diagn Res: JCDR. 2015 Dec; 9(12): ZE01. doi: 10.7860/ JCDR/2015/14275.6874

5. Sitam S, Hidajat NN, Arifin AZ. Panoramic Radiograph Analysis of Trabeculae, Cortical and Radius of Condyle Head in Post Menopausal Women. J Int Dent Med Res 2017; 10(1): 145-150.

6. Gudbjornsson B, Juliusson UI, Gudjonsson FV. Prevalence of long term steroid treatment and the frequency of decision making to prevent steroid induced osteoporosis in daily clinical practice. Ann Rheum Dis. 2002 Jan 1;61(1):32-36.

7. O'Keefe RJ, Mao J. Bone tissue engineering and regeneration: from discovery to the clinic-an overview. Tissue Eng Part B Rev. 2011 Oct 17;17(6):389-392. doi: 10.1089/ten. TEB.2011.0475. Epub 2011 Oct 19. 
8. Jafarian M, Eslaminejad MB, Khojasteh A, et al. Marrow-derived mesenchymal stem cellsdirected bone regeneration in the dog mandible: a comparison between biphasic calcium phosphate and natural bone mineral. Oral Surg Oral Med Oral Pathol Oral Radiol Endod. 2008 May 1; 105(5): e14-24. doi: 10.1016/j.tripleo.2008.01.010.

9. De Schauwer C, Van de Walle GR, Van Soom A, Meyer E. Mesenchymal stem cell therapy in horses: useful beyond orthopedic injuries?. Vet Q. 2013 Dec 1;33(4):234-241. doi: 10.1080/01652176.2013.800250. Epub 2013 May 22.

10. Oryan A, Alidadi S, Bigham-Sadegh A, Moshiri A, Kamali A. Effectiveness of tissue engineered chitosan-gelatin composite scaffold loaded with human platelet gel in regeneration of critical sized radial bone defect in rat. J Control Release. 2017 May 28;254:65-74. http://doi.org/10.1016/j.jconrel.2017.03.040

11. Spees JL, Lee RH, Gregory CA. Mechanisms of mesenchymal stem/stromal cell function. Stem Cell Res. Ther. 2016; 7(1):125. doi: 10.1186/s13287-016-0363-7.

12. Ratajczak J, Wysoczynski M, Hayek F, Janowska-Wieczorek A, Ratajczak MZ. Membrane-derived microvesicles: important and underappreciated mediators of cell-to-cell communication. Leukemia. 2006 Sep;20(9):1487. doi: 10.1038/sj.leu.2404296

13. Raposo G, Stoorvogel W. Extracellular vesicles: exosomes, microvesicles, and friends. J Cell Biol. 2013 Feb 18;200(4):373-83. doi: $10.1083 /$ jcb.201211138

14. Chen Y, Li G, Liu ML. Microvesicles as emerging biomarkers and therapeutic targets in cardiometabolic diseases. Genomics, proteomics \& bioinformatics. 2018 Feb 1;16(1):50-62. doi: 10.1016/j.gpb.2017.03.006. Epub 2018 Feb 17.

15. Ezzat BA, Abbass MM. The ability of $\mathrm{H} 1$ or $\mathrm{H}$ 2 receptor antagonists or their combination in counteracting the glucocorticoid-induced alveolar bone loss in rats. J Oral Pathol Med. 2014 Feb;43(2):148-156. doi: 10.1111/jop.12104. Epub 2013 Jul 12.

16. Chappard D, Josselin N, Rouge-Maillart C, Legrand E, Baslé MF, Audran M. Bone microarchitecture in males with corticosteroid-induced osteoporosis. Osteoporos Int. 2007 Apr 1;18(4):487-94.

17. Jia J, Yao W, Guan M, et al. Glucocorticoid dose determines osteocyte cell fate. FASEB J. 2011 Oct;25(10):3366-76. doi: 10.1096/fj.11-182519. Epub 2011 Jun 24.
18. McClung $M$, Harris ST, Miller PD, et al Bisphosphonate therapy for osteoporosis: benefits, risks, and drug holiday. Am J Med. 2013 Jan;126(1):13-20. doi: 10.1016/j. amjmed.2012.06.023. Epub 2012 Nov 20.

19. Cevidanes LH, Walker D, Schilling J, et al. 3D osteoarthritic changes in TMJ condylar morphology correlates with specific systemic and local biomarkers of disease. Osteoarthritis Cartilage. 2014 Oct 1;22(10):1657-1667. doi: 10.1016/j.joca.2014.06.014.

20. Turner AS. Animal models of osteoporosis necessity and limitations. Eur Cell Mater. 2001 Jun 22;1:66-81. PMID: 14562261.

21. Porto GG1, Vasconcelos BC, Andrade ES, SilvaJunior VA. Comparison between human and rat TMJ: anatomic and histopathologic features. Acta Cir Bras. 2010 Jun;25(3):290-293.

22. Lelovas PP, Xanthos TT, Thoma SE, Lyritis GP , Dontas IA: The Laboratory Rat as an Animal Model for Osteoporosis Research. Comp Med. 2008 Oct; 58(5): 424-430

23. Weinstein RS. The pathogenesis of glucocorticoidinduced osteoporosis. Clin Exper Rheumatology. 2000 Sep 1;18(5; SUPP/21):S-35.

24. Lane NE, Yao W, Balooch M, et al. Glucocorticoidtreated mice have localized changes in trabecular bone material properties and osteocyte lacunar size that are not observed in placebo-treated or estrogen-deficient mice. J Bone Miner Res. 2006 Mar;21(3):466-476. doi: 10.1359/JBMR.051103

25. Liu Y, Porta A, Peng X, et al. Prevention of glucocorticoid-induced apoptosis in osteocytes and osteoblasts by calbindin-D28k. J Bone Miner Res. 2004 Mar;19(3):479-490. doi: 10.1359/ JBMR.0301242

26. Canalis E. Mechanisms of glucocorticoid action in bone. Curr Osteoporos Rep. 2005 Sep;3(3):98102. PMID: 16131429

27. Hofbauer LC, Schoppet M. Clinical implications of the osteoprotegerin/RANKL/RANK system for bone and vascular diseases. JAMA. $2004 \mathrm{Jul}$ 28;292(4):490-495. doi: 10.1001/jama.292.4.490

28. Dovio A, Perazzolo L, Saba L, et al. Highdose glucocorticoids increase serum levels of soluble IL-6 receptor alpha and its ratio to soluble gp130: an additional mechanism for early increased bone resorption. Eur J Endocrinol. 2006 May;154(5):745-751. doi: 10.1530/eje.1.02147

29. Wang T, Yu X, He C. Pro-inflammatory cytokines: cellular and molecular drug targets for glucocorticoid-induced-osteoporosis via osteocyte. Curr Drug Targets. 2019;20(1):1-15. doi: 10.2174/ 1389450119666180405094046. 
30. Jabbar S, Drury J, Fordham JN, Datta HK, Francis RM, Tuck SP. Osteoprotegerin, RANKL and bone turnover in postmenopausal osteoporosis. J Clin Pathol. 2011 Apr;64(4):354-357. doi: 10.1136/ jcp.2010.086595.

31. Kiernan J, Hu S, Grynpas MD, Davies JE, Stanford WL. Systemic mesenchymal stromal cell transplantation prevents functional bone loss in a mouse model of age-related osteoporosis. Stem Cells Transl Med. 2016 May;5(5):683-93. doi: 10.5966/sctm.2015-0231.

32. Ocarino NdeM, Boeloni JN, Jorgetti V, Gomes DA, Goes AM, Serakides R. Intra-bone marrow injection of mesenchymal stem cells improves the femur bone mass of osteoporotic female rats. Connect Tissue Res. 2010 Dec;51(6):426-33. doi: $10.3109 / 03008201003597049$.

33. Akbar MA, LuY, ElshikhaAS, et al. Transplantation of adipose tissue-derived mesenchymal stem cell (ATMSC) expressing alpha-1 antitrypsin reduces bone loss in ovariectomized osteoporosis mice. Hum Gene Ther. 2017 Feb;28(2):179-189. doi: 10.1089/hum.2016.069.

34. Zhang HC, Liu XB, Huang S, et al. Microvesicles derived from human umbilical cord mesenchymal stem cells stimulated by hypoxia promote angiogenesis both in vitro and in vivo. Stem Cells Dev. 2012 Dec 10;21(18):3289-97. doi: 10.1089/ scd.2012.0095.
35. Zhang J, Liu X, Li H, et al. Exosomes/tricalcium phosphate combination scaffolds can enhance bone regeneration by activating the PI3K/Akt signaling pathway. Stem Cell Res Ther. 2016 Sep 20;7(1):136. doi: 10.1186/s13287-016-0391-3.

36. Wei J, Li H, Wang $\mathrm{S}$, et al. let-7 enhances osteogenesis and bone formation while repressing adipogenesis of human stromal/ mesenchymal stem cells by regulating HMGA2. Stem Cells Dev. 2014 Jul 1;23(13):1452-63. doi: 10.1089/ scd.2013.0600.

37. Zhu X, Yu J, Du J, Zhong G, Qiao L, Lin J. Lnc RNA HOXA-AS2 positively regulates osteogenesis of mesenchymal stem cells through inactivating NF- $\kappa \mathrm{B}$ signalling. J Cell Mol Med. 2019 Feb;23(2):1325-1332. doi: 10.1111/ jemm.14034.

38. Chen X, Liang H, Zhang J, Zen K, Zhang CY. Horizontal transfer of microRNAs: molecular mechanisms and clinical applications. Protein Cell. 2012 Jan;3(1):28-37. doi: 10.1007/s13238012-2003-z.

39. Panagiotou N, Wayne Davies R, Selman C, Shiels PG. Microvesicles as vehicles for tissue regeneration: changing of the guards. Curr Pathobiol Rep. 2016;4(4):181-187. doi: 10.1007/ s40139-016-0115-5. 